\title{
Characterization of Outer Membrane Vesicle Release in Vibrio vulnificus.
}

\author{
Cheri Hampton ${ }^{1}$, Ricardo C. Guerrero-Ferreira ${ }^{2}$, Hong Yi $^{3}$ and Elizabeth R. Wright ${ }^{1,3}$ \\ ${ }^{1 .}$ Emory University School of Medicine, Division of Pediatric Infectious Disease, and Children's \\ Healthcare of Atlanta, Atlanta, Georgia \\ 2. Laboratory of Structural Biology and Biophysics, Institute of Physics of Biological Systems, École \\ Polytechnique Fédérale de Lausanne, Switzerland. \\ 3. Robert P. Apkarian Integrated Electron Microscopy Core, Emory University, Atlanta, Georgia
}

The production of outer membrane vesicles (OMVs) by Gram-negative bacteria is an active process that takes place when a section of the outer membrane bulges off to form periplasm-containing vesicles without causing bacterial lysis. OMVs directly influence bacteria-host interaction and pathogenesis through their ability to modulate immune responses, contribute to biofilm formation, and deliver toxins and other virulence factors to host cells [1]. The ability of OMVs to bind to eukaryotic membranes makes them potential delivery vehicles for antibiotics and candidate vaccines against pathogens such as Vibrio cholerae, indicating their significant biotechnological potential [2]. OMVs have also been recognized to be important components of bacterial biofilms. $V$. vulnificus is a Gram-negative bacterium which colonizes filter-feeding shellfish. This opportunistic pathogen is responsible for $95 \%$ of seafoodrelated deaths in the United States and is categorized as a category B biodefense priority pathogen by the National Institutes of Allergies and Infectious Diseases [3]. Virulence in V. vulnificus is regulated by structural components such as cellular appendages (flagella and pili) and the capsular polysaccharide. Even though their contribution to bacterial pathogenesis is widely recognized, the mechanism of OMV production is not yet understood.

Here, we use multiple TEM imaging techniques to characterize the unusual secretion and spatial arrangement of OMVs. We present further cryo-electron tomography data [4] (Fig. 1) of OMV release and distribution in Vibrio vulnificus and several of its mutant forms in order to link these results to known differences in virulence. Because of the low contrast of cryo TEM, the capsular polysaccharide remained obscure. In order to visualize the role of the capsular polysaccharide in providing this unique spatial arrangement we employ a high pressure freezing technique, self pressurized rapid freezing (SPRF) [5]. For cryo EM and cryo ET, V. vulnificus was grown in LB broth at 30 degrees $\mathrm{C}$ with shaking at $250 \mathrm{rpm}$ overnight, then diluted into fresh LB and grown and additional 4 hours. Bacteria were applied to copper 200 mesh grids with R2/1 Quantifoil carbon supports and plunge frozen into liquid ethane. For SPRF, colonies from a fresh LB plate were scraped into a paste and thinned with enough LB broth to facilitate pipetting into a $16 \mathrm{~mm}$ long and $0.65 \mathrm{~mm}$ o.d. copper tube. After crimping the ends closed, the tube was manually plunged into liquid propane. Tubes were sectioned and freezesubstituted.

Both cryo EM and cryo ET reveal a regular arrangement of OMVs spaced approximately $100 \mathrm{~nm}$ from the outer membrane and in successive rings with $200 \mathrm{~nm}$ spacing. We do not observe this discrete spacing in reduced virulence mutants lacking the capsular polysaccharide. We were able to visualize the capsular polysaccharide using the SPRF technique, and observe that the capsule extends in a radius around the outer membrane a distance of $\sim 100 \mathrm{~nm}$, and that the individual OMVs retained these $100 \mathrm{~nm}$ polysaccharide chains which remain joined at the distal end, creating $200 \mathrm{~nm}$ spacing between the outer membrane and OMVs, as well as between rows of OMVs (Fig. 2). We hypothesize that the capsular polysaccharide has a definitive role in OMV distribution and virulence in $V$. vulnificus. 


\section{References:}

[1] A Kulp and MJ Kuehn. Annual Review of Microbiology 64 (2010), p. 163-184.

[2] S Schild, EJ Nelson, AL Bishop, and A Camilli. Infection and Immunity 77 (2009), p. 472-484.

[3] MS Strom and RN Paranjpye. Microbes Infection 2 (2000), p. 177-188.

[4] R Guerrero-Ferreira, G Williams, and ER Wright. Microscopy and Microanalysis 17 (2011), p. 142143.

[5] JLM Leunissen and H Yi. Journal of Microscopy 235, pt 1 (2009), p. 25-35.

[6] This research was supported by funds from Emory University, Children's Healthcare of Atlanta, the Emory Center for AIDS Research, the Georgia Research Alliance, Human Frontiers Science Program, National Institutes of Health (1R01GM104540), and the National Science Foundation (0923395) to E.R.W, and S10 RR025679 to P.W.S. All EM data was collected at the Emory University Robert P. Apkarian Integrated Electron Microscopy Core.

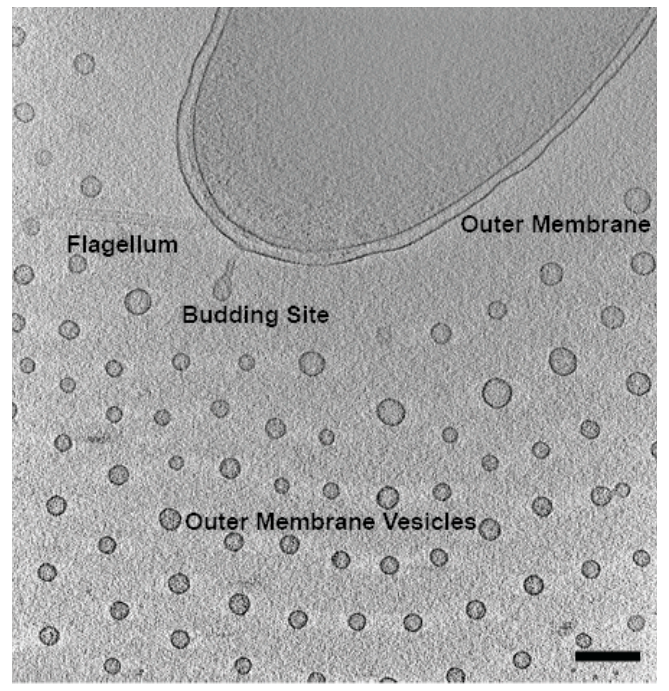

Figure 1. Cryo ET slice of plunge-frozen $V$. vulnificus producing outer membrane vesicles. Scale bar $200 \mathrm{~nm}$.

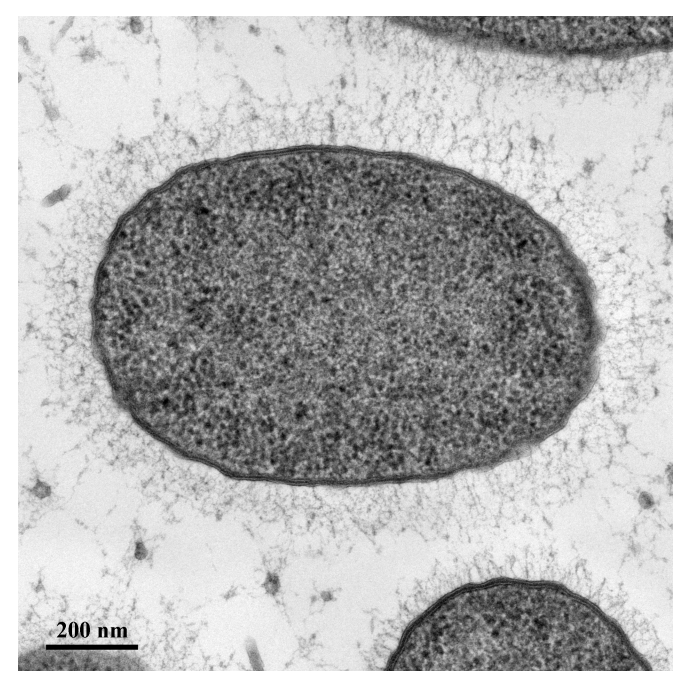

Figure 2. SPRF, freeze-substituted section of $V$. vulnificus reveals the capsular polysaccharide chains extending from the outer membrane and attached to surrounding OMVs. Scale bar $200 \mathrm{~nm}$. 